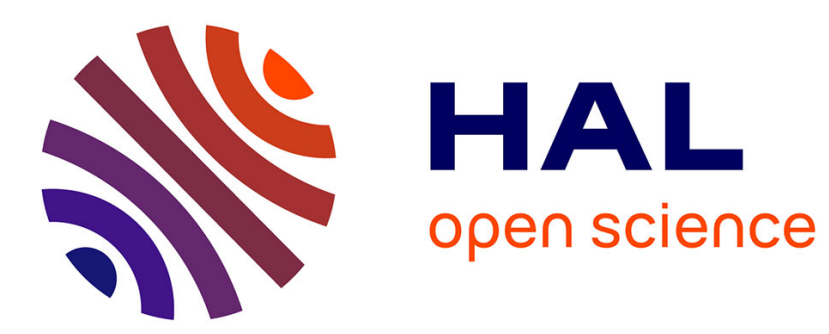

\title{
La fiscalité environnementale en France peut-elle devenir réellement écologique?
}

\author{
Mireille Chiroleu-Assouline
}

\section{To cite this version:}

Mireille Chiroleu-Assouline. La fiscalité environnementale en France peut-elle devenir réellement écologique?: État des lieux et conditions d'acceptabilité. Revue de l'OFCE, 2015, 139, pp.129-165. 10.3917/reof.139.0129 . halshs-01199478

\section{HAL Id: halshs-01199478 https://shs.hal.science/halshs-01199478}

Submitted on 15 Sep 2015

HAL is a multi-disciplinary open access archive for the deposit and dissemination of scientific research documents, whether they are published or not. The documents may come from teaching and research institutions in France or abroad, or from public or private research centers.
L'archive ouverte pluridisciplinaire HAL, est destinée au dépôt et à la diffusion de documents scientifiques de niveau recherche, publiés ou non, émanant des établissements d'enseignement et de recherche français ou étrangers, des laboratoires publics ou privés. 


\section{LA FISCALITÉ ENVIRONNEMENTALE EN FRANCE PEUT-ELLE DEVENIR RÉELLEMENT ÉCOLOGIQUE ?}

État des lieux et conditions d'acceptabilité

Mireille Chiroleu-Assouline

OFCE | « Revue de l'OFCE »

2015/3 N 139 | pages 129 à 165

ISSN 1265-9576

ISBN 9782312033778

Article disponible en ligne à l'adresse :

http://www.cairn.info/revue-de-1-ofce-2015-3-page-129.htm

\section{!Pour citer cet article :}

Mireille Chiroleu-Assouline, « La fiscalité environnementale en France peut-elle devenir réellement écologique ? État des lieux et conditions d'acceptabilité », Revue de l'OFCE 2015/3 $\left(\mathrm{N}^{\circ} 139\right)$, p. 129-165.

DOI 10.3917/reof.139.0129

Distribution électronique Cairn.info pour OFCE.

(C) OFCE. Tous droits réservés pour tous pays.

La reproduction ou représentation de cet article, notamment par photocopie, n'est autorisée que dans les limites des conditions générales d'utilisation du site ou, le cas échéant, des conditions générales de la licence souscrite par votre établissement. Toute autre reproduction ou représentation, en tout ou partie, sous quelque forme et de quelque manière que ce soit, est interdite sauf accord préalable et écrit de l'éditeur, en dehors des cas prévus par la législation en vigueur en France. Il est précisé que son stockage dans une base de données est également interdit. 


\section{Partie 3}

\section{FISCALITÉ ET TRANSITION ÉCOLOGIQUE}

La fiscalité environnementale en France peut-elle devenir réellement écologique ? État des lieux et conditions d'acceptabilité . . . . . 131 Mireille Chiroleu-Assouline

La taxe-carbone : une idée toujours d'avenir si... . . . . . . 167 Jean-Charles Hourcade 


\title{
LA FISCALITÉ ENVIRONNEMENTALE EN FRANCE PEUT-ELLE DEVENIR RÉELLEMENT ÉCOLOGIQUE ? ÉTAT DES LIEUX ET CONDITIONS D'ACCEPTABILITÉ
}

\author{
Mireille Chiroleu-Assouline \\ Paris School of Economics \\ Université Paris 1 Panthéon-Sorbonne \\ INRA-UMR Économie Publique
}

Actuellement, la fiscalité environnementale française répond moins à une finalité écologique qu'à un objectif plus traditionnel de fiscalité de rendement. Les signes manifestes de cette inadéquation sont la très grande part prise par la fiscalité de l'énergie et le faible niveau de la plupart des taux de taxe, qui souvent ne frappent qu'implicitement les produits polluants. Réformer la fiscalité française supposerait de la «verdir » dans son ensemble en appliquant des taux de taxes en relation avec les dommages marginaux. La réussite de la réforme et son acceptation par les contribuables sont conditionnées par le mécanisme de redistribution associé, les efforts de pédagogie et d'information, la transparence mais aussi, paradoxalement, par l'audace des mesures prises.

Mots clés : fiscalité, écotaxe, contribution climat-énergie, double dividende, progressivité de l'impôt.

$\mathrm{E}_{\mathrm{n}}$

septembre 2012 s'est tenue à Paris la première Conférence environnementale pour la transition écologique ${ }^{1}$, ouverte par le président François Hollande qui y a affiché l'objectif de faire de la France la nation de l'excellence environnementale. Il s'agit de réduire la pression sur les ressources naturelles épuisables (eau, matières premières, énergies fossiles), de réduire les atteintes à la

1. Cette conférence s'est depuis tenue chaque année, définissant une nouvelle feuille de route pour la transition écologique. En 2014, la fiscalité environnementale ne faisait pas partie des thèmes explicitement abordés. 
nature, de lutter contre les activités polluantes et le changement climatique. L'une des composantes incontournables de la transition écologique est ainsi la transition énergétique, autrement dit le passage d'une société fondée sur la consommation abondante d'énergies fossiles à une société plus sobre en énergie et faiblement carbonée, grâce en particulier au développement des énergies renouvelables.

La fiscalité écologique pourrait être l'un des éléments essentiels des politiques mises en œuvre pour réaliser ces objectifs. Elle figurait en effet parmi les instruments de financement mis en avant par la Feuille de route 2012 pour la transition écologique. Un comité pour la fiscalité écologique a d'ailleurs été créé en décembre $2012^{2}$, avec pour ambition de constituer un dispositif permanent de concertation et d'évaluation de la fiscalité écologique, et pour mission de formuler un avis sur les mesures fiscales écologiques proposées par le gouvernement et de faire des propositions en la matière.

Où en est-on, début 2015, dans ce domaine de la fiscalité écologique ? L'objectif de cet article est d'établir un état des lieux de la mise en place d'une fiscalité écologique cohérente et efficace en France ainsi que d'éclairer les raisons des difficultés rencontrées et les conditions de son acceptation par les différents acteurs de la vie économique. La première partie reviendra sur la définition de la fiscalité écologique, qui suggère de procéder à une distinction sémantique quant à l'appellation écologique ou environnementale de ce type de fiscalité. Dans un deuxième temps, nous montrerons que la situation actuelle de la France en termes de fiscalité environnementale est loin de répondre aux objectifs affichés. Ceci nous amènera à énoncer dans la troisième partie des conditions nécessaires pour la mise en place d'une fiscalité écologique à la fois efficace et acceptable socialement.

\section{Fiscalité environnementale ou écologique?}

Selon une définition commune aux différents instituts de statistiques ou d'étude, français et européens (CGDD, Eurostat, OCDE), la fiscalité environnementale recouvre d'une part toutes les taxes

2. Il a rendu un rapport d'étape en juillet 2013 et publié plusieurs avis. De tels comités ont été créés dans d'autres pays comme le Portugal ou le Canada (Withana et al., 2014). 
portant sur des produits ou des actifs ayant des effets nuisant à la qualité de l'environnement, comme les taxes liées à la consommation d'énergie, les taxes sur les véhicules, la taxation des pollutions et des déchets, la taxation de la consommation d'eau; et d'autre part les dépenses fiscales favorisant le développement durable (dispositions législatives ou réglementaires dont la mise en œuvre entraîne pour l'État une perte de recettes par rapport à ce qui serait résulté de l'application des principes généraux du droit fiscal français) comme les exonérations ou crédits d'impôt. Le dispositif de bonus-malus mis en place pour l'immatriculation des véhicules automobiles neufs est une combinaison de taxe-subvention qui fait partie de cet ensemble. La fiscalité environnementale est donc fondamentalement définie par son assiette et non par le mode d'utilisation de ses recettes, ni par l'intention ayant présidé à sa mise en place.

En 2012, les recettes fiscales environnementales se sont élevées pour la France à 37,2 milliards d'euros, soit 1,83\% du PIB et 4,08 \% du total des prélèvements obligatoires (d'après Eurostat).

Il est d'ailleurs intéressant de rapporter le montant des recettes fiscales environnementales au montant des dépenses de protection de l'environnement, définies comme "l'effort financier entrepris par les différentes composantes de la société - administrations publiques, entreprises privées et ménages - pour prévenir, réduire ou supprimer les dégradations causées à l'environnement " (CGDD, 2014a). Or celles-ci s'élèvent au total à 47,5 milliards d'euros en 2012, soit 2,27\% du $\mathrm{PIB}$, tous financeurs confondus. Les administrations publiques financent 31,8\% de ces dépenses, ce qui correspond à 15,1 milliards d'euros. Il est donc clair que les recettes fiscales environnementales ne financent pas ces dépenses spécifiques mais qu'elles abondent le budget de l'État conformément à la règle de finances publiques de non affectation des recettes fiscales ${ }^{3}$.

À cette définition de la fiscalité environnementale par l'assiette se superpose une définition par la finalité assignée par la théorie économique à la fiscalité écologique. En effet, l'objectif d'une fiscalité écologique est d'internaliser les externalités, le plus souvent

3. Comme toute règle, celle-ci admet des exceptions : par exemple, une hausse de 2 centimes de la TIC sur le gazole a été votée en octobre 2014 pour financer l'Agence de financement des infrastructures de transport (Atfit) en compensation de l'abandon de l'écotaxe poids-lourds. 
négatives. Selon le principe de la taxation pigouvienne (Pigou, 1920), les émissions polluantes doivent être taxées à un taux unitaire égal au dommage marginal qu'elles provoquent. De la sorte, conformément au principe pollueur-payeur, les agents privés à la source de l'externalité subissent, en sus de leur coût privé de production du bien qui provoque la pollution, le coût externe imposé aux victimes de la pollution. Ceci les amène à prendre leurs décisions en fonction du coût social total de la pollution, internalisant ainsi le coût externe ${ }^{4}$. C'est ce mécanisme qui est couramment dénommé signal-prix : en effet, l'augmentation du prix du bien polluant due à la taxe provoque une hausse de son prix relatif par rapport à d'autres biens, en particulier moins polluants. Ce signal incite alors les acheteurs, consommateurs ou industriels, à remplacer des produits polluants par d'autres non polluants, ou à adopter des technologies moins polluantes. Un signal-prix stable et pérenne stimule également l'innovation en poussant les industriels à chercher des solutions moins polluantes pour réduire leurs coûts de production ou à proposer des produits moins polluants pour profiter des opportunités de marché ainsi ouvertes par la régulation environnementale. Modifiant ainsi les prix relatifs, toute taxe écologique est par nature distordante - à l'instar de toute taxe indirecte - mais cette distorsion est souhaitée dans la mesure où elle vient corriger une défaillance de marché. En effet, la fiscalité écologique se justifie par sa nature incitative (Chiroleu-Assouline, 2011).

Comme on le voit, la fiscalité écologique est a priori environnementale et il serait cohérent que la fiscalité environnementale soit écologique. Or l'examen de la situation en France montre que c'est encore loin d'être le cas, même si, dans la pratique, les deux termes sont employés indifféremment. La fiscalité est si sophistiquée que la distinction sémantique introduite ici ne doit pas être réduite à la distinction entre impôt de rendement et impôt incitatif, ni à une différence entre une définition selon l'assiette et une définition selon les taux.

La Feuille de route pour la transition écologique adoptée en 2012 reconnaissait le rôle d'incitation de comportements vertueux et de dissuasion de comportements néfastes que la fiscalité écologique est à même de jouer pour réduire les émissions de gaz à effet

4. Voir Baumol et Oates (1988) ou Beaumais et Chiroleu-Assouline (2002). 
de serre, les pollutions diverses et l'utilisation des ressources naturelles. Elle établissait en outre le constat que «des instruments fiscaux existent dans notre pays mais demeurent incomplets ou perfectibles » et que «de fait, la mise en place d'une fiscalité environnementale reste largement inaboutie en France». En définissant des voies de réforme, le gouvernement y affichait l'objectif «de faire converger notre pays vers la moyenne de l'Union européenne ", tout en mettant au centre de l'analyse le rôle de signal-prix et les arbitrages à effectuer pour tenir compte des problèmes de compétitivité, de redistribution et de précarité. En insistant sur la nécessité de ne pas la considérer comme une source de rendement pour les finances publiques, le document n'assigne qu'une place secondaire à la fiscalité écologique parmi les différents instruments de financement possibles pour la transition écologique. Tous les grands principes figurent dans cette feuille de route : réformer la fiscalité écologique, utiliser son effet-signal plutôt que son potentiel d'instrument de financement, rapprocher taux de taxation des dommages marginaux, etc. La stratégie affichée dès lors par le gouvernement est celle d'une réforme d'ampleur du système français de prélèvements obligatoires qui, néanmoins, fin 2014, n'a pas encore été mise en œuvre.

\section{La fiscalité environnementale en France aujourd'hui}

Le système fiscal français accorde une place peu significative à la fiscalité environnementale : à un niveau de 1,83\% du PIB en 2012, le poids des recettes fiscales environnementales est faible et il est loin d'augmenter conformément aux objectifs affichés puisque, depuis 2001, il est même en légère diminution (Eurostat). Il place la France à la $24^{\mathrm{e}}$ place parmi les 28 pays de l'Union européenne, pour l'ensemble des recettes comme pour celles tirées des taxes sur l'énergie qui en constituent la plus grande part ( $80 \%$, soit 1,47\% $\mathrm{du}$ PIB). Les recettes fiscales dues aux taxes sur les transports et à celles sur la pollution et les ressources représentent respectivement $0,24 \%$ du PIB (23 ${ }^{\mathrm{e}}$ place/UE28) et $0,13 \%$ du PIB (11 $\left.\mathrm{e} / \mathrm{UE} 28\right)$. Le graphique 1 met en évidence le faible poids de ces recettes fiscales par rapport au PIB en comparaison avec la moyenne des pays de l'Union européenne à 28. 
Graphique 1. Poids des recettes fiscales environnementales, en 2012

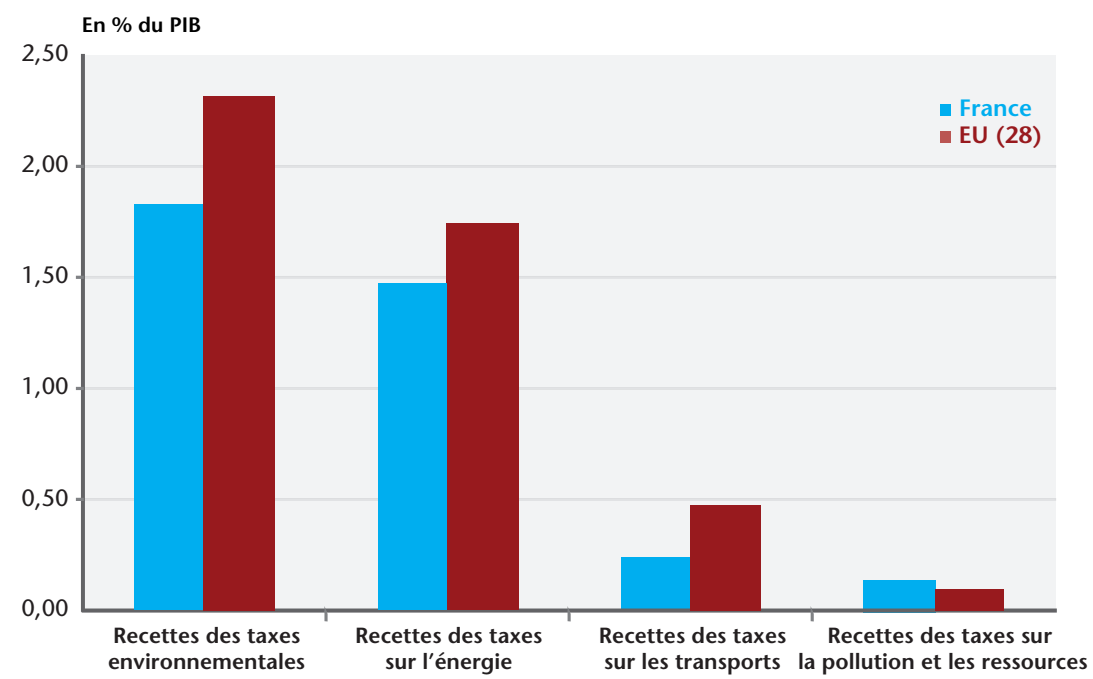

Source : EUROSTAT $:$ http://ec.europa.eu/eurostat/tgm/table.do?tab=table\&init=1\&language=en\&pcode=ten00065 \&plugin $=1$

Il est important de souligner qu'en 2012, le verdissement du système fiscal français n'était pas engagé puisqu'au contraire les recettes des taxes environnementales ont progressé beaucoup moins vite que l'ensemble des prélèvements obligatoires depuis 1995 (graphique 2), leur part dans les prélèvements obligatoires décrochant ainsi encore davantage que leur poids mesuré par rapport au PIB et continûment, de 5,82 \% en 1995 à 4,08 \% en 2012 (Eurostat).

De même la fiscalité écologique ne tient pas une place marquante dans le financement de la transition écologique comme en témoigne le suivi de la mise en ouvre de la Feuille de route 2012 pour la transition écologique du ministère de l'Écologie, du développement durable et de l'énergie (MEDD, octobre 2014). Ce document renvoie aux travaux du CFE pour tout ce qui touche à la fiscalité ${ }^{5}$

5. Le CFE n'a plus été réuni depuis février 2014, et son président, Christian de Perthuis, a démissionné en octobre 2014, «découragé » et sous «l'impression que j'ai est que le verdissement de la fiscalité n'est pas une priorité gouvernementale » (Le Monde, 13/10/2014). Début février 2015, le comité a été rebaptisé Comité pour l'économie verte et a vu ses missions redéfinies et élargies. Il est maintenant chargé de la réflexion sur les "mécanismes de marché tels que les permis, les quotas, les certificats » ainsi que sur les « outils de mobilisation des financements, notamment privés, au bénéfice de la transition écologique et énergétique ». Cette évolution illustre clairement la dilution de l'intérêt porté à la fiscalité écologique dans la perspective plus large de trouver des modes de financement de la croissance verte. 
tandis que le chapitre consacré au financement de la transition ne mentionne que le renforcement de la dimension développement durable de l'AFD et des règles de passation des marchés publics.

Graphique 2. Évolution du total des prélèvements obligatoires et des recettes fiscales environnementales

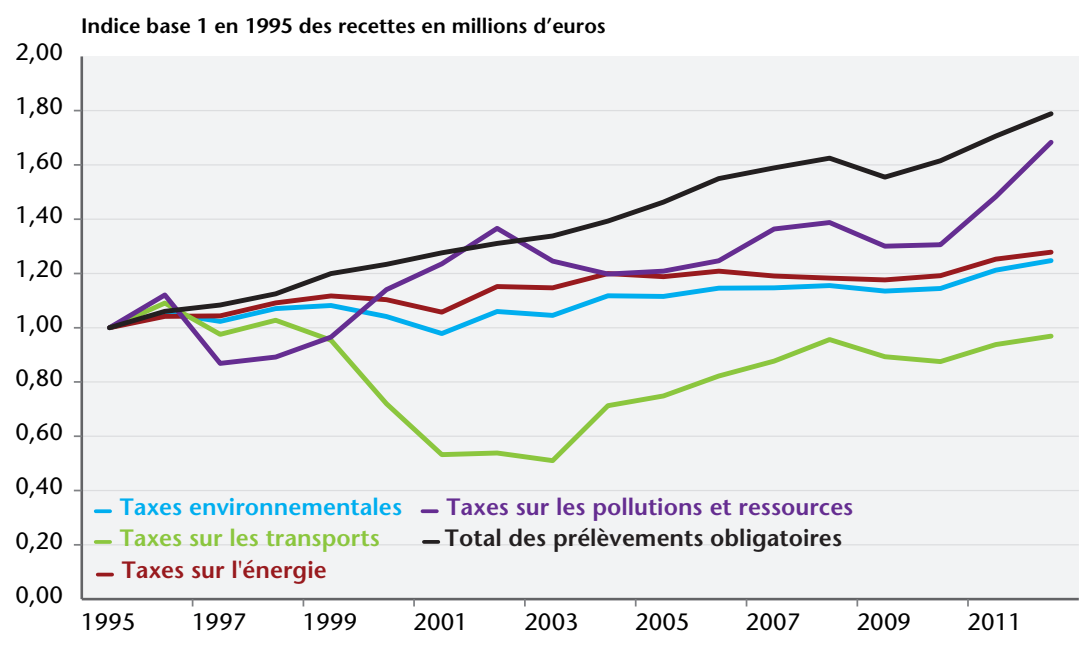

Source : EUROSTAT : http://epp.eurostat.ec.europa.eu/portal/page/portal/product_details/dataset?p_product_code= ENV_AC_TAX (dernière mise à jour : 25/11/2014).

La fiscalité environnementale comprend également les dépenses fiscales favorables à l'environnement, comme le crédit d'impôt au développement durable, ou d'autres subventions. Pour l'année 2011, les mesures de ce type étaient au nombre de 29 dans le système fiscal français pour un total d'environ 2,6 milliards d'euros, mais avec des montants très divers et souvent anecdotiques (CGDD, 2013), le crédit d'impôt développement durable représentant 1,95 milliard d'euros, soit dix fois plus que l'exonération de la taxe intérieure de consommation sur les biocarburants, la deuxième dépense par ordre d'importance ( $c f$ tableau 1 ).

Ces dépenses fiscales sont susceptibles de modifications plus rapides et d'ailleurs souvent moins conflictuelles que les recettes. Par exemple, le texte de la loi «sur la transition énergétique pour une croissance verte ", annoncée depuis 2012 et votée début 2015, ajoute un nouvel allégement fiscal de $30 \%$ pour les travaux de rénovation énergétique engagés entre le $1^{\mathrm{er}}$ septembre 2014 et le 31 décembre 2015, pour tenter d'atteindre l'objectif d'un million de 
logements thermiquement isolés par an fixé lors de la campagne présidentielle de 2012. Un chèque énergie est également mis en place pour les ménages modestes, pour remplacer à terme les tarifs sociaux existants sur le gaz et l'électricité, et pour contribuer à financer des travaux de rénovation thermique.

Tableau 1. Principales dépenses fiscales favorables à l'environnement

\begin{tabular}{|c|c|c|c|c|}
\hline Impôt & Mesure & $\begin{array}{l}\text { Bénéfi- } \\
\text { ciaires }\end{array}$ & $\begin{array}{l}\text { Chiffrage } \\
2011 \text { (M€) }\end{array}$ & $\begin{array}{l}\text { Année de } \\
\text { création }\end{array}$ \\
\hline IR & $\begin{array}{l}\text { Crédit d'impôt pour dépenses d'équipements de } \\
\text { I'habitation principale en faveur des économies } \\
\text { d'énergie et du développement durable (CIDD) }\end{array}$ & M & 1950 & 1999 \\
\hline TICPE & $\begin{array}{l}\text { Exonération plafonnée de taxe intérieure de } \\
\text { consommation pour les biocarburants }\end{array}$ & $E$ & 196 & 1995 \\
\hline ISF & $\begin{array}{l}\text { Exonérations partielle des bois et forêts et des parts } \\
\text { d'intérêts détenues dans un groupement forestier, } \\
\text { des biens ruraux loués par bail à long terme et des } \\
\text { parts de GFA }\end{array}$ & M & 52 & 1981 \\
\hline TFPB & $\begin{array}{l}\text { Dégrèvements sur la cotisation de taxe foncière sur } \\
\text { les propriétés bâties pour les organismes HLM et } \\
\text { les SEM à raison des travaux d'énergie (égal au } \\
\text { quart des dépenses) }\end{array}$ & $\mathrm{E}$ & 50 & 2005 \\
\hline IS & $\begin{array}{l}\text { Éco prêt à taux zéro (identifié comme crédit } \\
\text { d'impôt IS aux établissements de crédit) }\end{array}$ & M & 50 & 2008 \\
\hline TICPE & $\begin{array}{l}\text { Taux réduit de taxe intérieure de consommation } \\
\text { sur le GPL }\end{array}$ & $\mathrm{M}+\mathrm{E}$ & 41 & 2007 \\
\hline IR-IS & $\begin{array}{l}\text { Crédit d'impôt en faveur des entreprises agricoles } \\
\text { utilisant le mode de production biologique }\end{array}$ & $E$ & 33 & 2006 \\
\hline TVA & $\begin{array}{l}\text { Taux de } 5,5 \% \text { pour la fourniture par réseaux } \\
\text { d'énergie d'origine renouvelable }\end{array}$ & $E$ & 25 & 2006 \\
\hline
\end{tabular}

Source : CGDD (2013), La fiscalité environnementale en France - États des lieux.

Le bonus-malus écologique mis en place en 2008 à la suite du Grenelle de l'environnement a un statut particulier : le barème du bonus versé aux acquéreurs d'un véhicule neuf émettant peu de $\mathrm{CO}_{2}$ est d'ordre réglementaire tandis que celui du malus relève de la Loi de finances puisqu'il prend la forme d'une taxe additionnelle à la taxe sur les certificats d'immatriculation (carte grise), pour l'achat d'un véhicule émettant beaucoup de $\mathrm{CO}_{2}$. Le dispositif devait être équilibré financièrement mais dans la mesure où le barème influence fortement à la fois la demande et l'offre sur le marché des véhicules neufs, il est assez difficile de prévoir avec une précision suffisante ses recettes et dépenses. Structurellement déficitaire depuis 2008 où le différentiel à la charge de l'État (entre les bonus versés et les malus encaissés) était de +617 millions d'euros, 
il a été bénéficiaire en 2012, à la suite d'un durcissement du barème, pour atteindre - 102 millions d'euros, puis de nouveau +100 millions d'euros en 2013. En cas de déficit du dispositif, celuici apparaît dans le compte de dépense de protection de la qualité de l'air du point de vue monétaire (CGDD, 2014a).

\subsection{Une fiscalité dévoyée par la recherche du rendement plutôt que des incitations}

Au-delà de la constatation de cette faible place, force est de constater que, comme le souligne le Rapport d'étape du Comité pour la fiscalité écologique (CFE, 2013), la structure du système fiscal français reflète plus une logique de rendement fiscal que d'incitations écologiques. Il a en effet été constitué par additions et modifications successives d'impôts initialement mis en place comme des impôts de rendement, même s'ils sont aujourd'hui considérés comme supports de la fiscalité écologique.

Le premier signe du dévoiement de la fiscalité environnementale actuelle est que les quatre cinquièmes des recettes fiscales concernées proviennent des taxes sur l'énergie, la TICPE (Taxe intérieure sur la consommation de produits énergétiques, ex TIPP) rapportant à elle seule les deux tiers du produit.

D'un autre côté, les taxes à visée explicitement écologique que sont la TGAP (Taxe générale sur les activités polluantes) ou les taxes sur la pollution et les prélèvements de l'eau ne procurent que $6 \%$ du rendement total des taxes environnementales, soit $0,1 \%$ du PIB! La TGAP a pourtant un champ d'application très large puisqu'elle résulte du regroupement en 1999 de cinq taxes préexistantes touchant les déchets industriels spéciaux, le stockage des déchets ménagers et assimilés, la pollution atmosphérique, les huiles de base, l'atténuation des nuisances sonores au voisinage des aérodromes. Elle est maintenant due par toutes les entreprises dont l'activité ou les produits sont considérés comme polluants: déchets, émissions polluantes, huiles et préparations lubrifiantes, lessives, solvants, matériaux d'extraction, pesticides, produits chimiques,... De même, le poids des taxes sur les transports $(0,24 \%$ du PIB) est deux fois plus faible en France que dans la moyenne des pays européens $(0,47 \%)$. 
La raison originelle de la création de ces taxes sur l'énergie est davantage leur rendement que leur éventuel pouvoir incitatif à la réduction de la consommation d'énergie. En effet, l'énergie est un bien essentiel et sa demande est relativement inélastique : les produits énergétiques constituent donc une assiette s'érodant faiblement, ce qui assure la stabilité des recettes fiscales.

Or, ce qui fait la force d'un impôt de rendement fait la faiblesse d'un impôt incitatif : dans le premier cas, il suffit d'un taux faible appliqué à une assiette large pour garantir des recettes importantes alors que dans l'autre, seules des hausses importantes des taux peuvent réduire significativement la consommation.

Le deuxième signe du caractère peu écologique, car peu incitatif, de la fiscalité environnementale actuelle est justement la faiblesse de la plupart des taux de taxe. En effet, à l'exception des taux de taxe sur les carburants qui sont à un niveau important (OCDE, 2013), les taux de taxe effectifs apparents sur les différents produits énergétiques selon leur utilisation ou sur les polluants les plus courants de l'air ou de l'eau sont faibles, peu cohérents entre eux et très différents de ceux pratiqués dans les autres pays européens. Il y a donc une forte présomption d'absence d'efficacité économique et de non-internalisation des dommages marginaux.

Le graphique 3 ci-dessous montre les taux de taxe effectifs apparents sur le dioxyde de carbone émis par les sources d'énergie selon leur utilisation. Ce sont des taux de taxe implicites sur le carbone car même si leur assiette n'est pas explicitement le contenu en carbone de chaque énergie, on peut utiliser les taux d'émission pour calculer des taux de taxe moyens sur les émissions de $\mathrm{CO}_{2}$. Il est frappant de constater que seule la taxe affectant les carburants utilisés pour le transport routier est très élevée : 182 euros en moyenne, selon les calculs de l'OCDE (2013) avec des différences très marquées entre types de carburant qui n'ont guère varié depuis l'étude de l'ADEME (2009) évaluant le taux apparent d'imposition $2007 \mathrm{du}$ supercarburant sans plomb à $265 € / \mathrm{t} \mathrm{CO}_{2}$ et celui du gazole à $158 € / \mathrm{t} \mathrm{CO}_{2}$. Ces niveaux élevés doivent sans doute moins à Pigou qu'à Ramsey (1927) dont la théorie de la taxation indirecte optimale montre qu'il est plus efficace, pour prélever un revenu fiscal donné de différencier les taux de taxation des biens en relation inverse de l'élasticité-prix de la demande. 
Graphique 3. Taux de taxe effectifs apparents dans I'UE-28

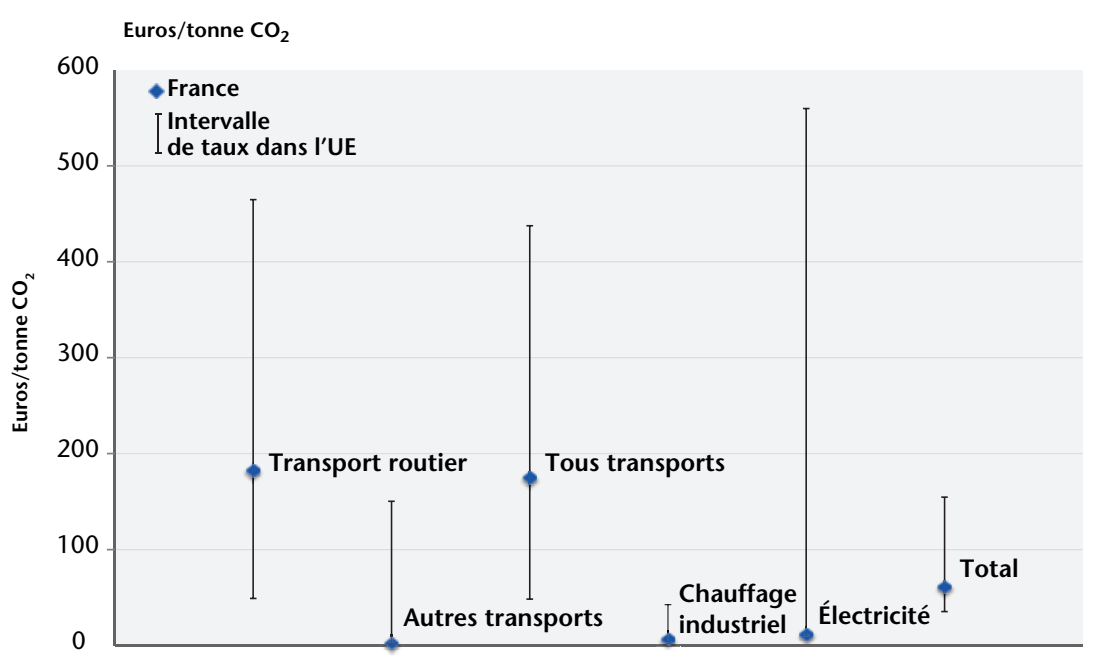

Source : OCDE (2013), Taxing Energy Use.

Les taux apparents de taxation du dioxyde de carbone pratiqués en France sont néanmoins tous dans le bas de l'intervalle des taux des autres pays européens, en tout état de cause très inférieurs à ceux pratiqués en Allemagne (Cour des comptes, 2011). Le charbon est l'énergie fossile la plus émettrice de $\mathrm{CO}_{2}$ et pourtant la moins taxée ${ }^{6}$. Les autres carburants (kérosène, LPG) sont taxés à des taux encore plus faibles que l'essence sans plomb et le diesel, voire exonérés (gaz naturel).

Toutes les sources s'accordent pour constater que le poids de la fiscalité énergétique en France est inférieur à la moyenne européenne essentiellement parce que les combustibles fossiles sont sous-taxés (au contraire des carburants), l'écart s'étant creusé depuis l'adoption d'une taxe carbone par la Suède, le Danemark, le Royaume-Uni et la Finlande. Mais ce n'est pas l'unique raison. En effet, malgré son taux nettement plus élevé que pour les autres énergies, le taux de taxe sur l'essence sans plomb n'a été ajusté en termes nominaux que de façon minimale depuis 2004 et son montant en valeur a été érodé par l'inflation (Cour des comptes, 2011). L'absence d'indexation est à l'origine d'une perte de recettes annuelles estimée à environ 1,3 milliard d'euros en ce qui

6. En revanche, les centrales à charbon sont assujetties au marché européen des quotas de $\mathrm{CO}_{2}$. 
concerne l'essence et de 1,6 milliard d'euros pour le gazole, par rapport à ce qui aurait pu être obtenu si ces taxes avaient été maintenues au même niveau en termes réels. Du point de vue de la Cour des comptes, il est vraisemblable que le problème est dénoncé davantage en raison de la perte de rendement que de la perte d'efficacité écologique.

En ce qui concerne les taux de la TGAP visant les polluants atmosphériques $\left(\mathrm{NO}_{2}, \mathrm{NO}_{\mathrm{x}}\right.$, VOCs, $\left.\mathrm{SO}_{\mathrm{x}}, \mathrm{PM}_{2.5}\right)$, force est de constater qu'ils sont eux-mêmes très faibles, avec un maximum à $167 € /$ tonne pour les oxydes d'azote. Ils sont ainsi considérablement plus faibles que les coûts externes de la pollution de l'air, de l'ordre de plusieurs milliers d'euros par tonne de polluant, selon les estimations pour la France disponibles grâce à diverses études comme RICARDO-AEA (2014) ou EEA (2013) reposant sur les évaluations du Handbook on estimation of external costs in the transport sector (CE Delft/INFRAS, 2008).

D'autres taux ont été arbitrairement réduits comme celui de la taxe à l'essieu qui pèse sur les véhicules de plus de 12 tonnes, abaissé en 2009 pour préparer l'introduction de l'écotaxe poidslourds prévue initialement pour janvier 2014. Malgré le report sine die puis l'abandon de celle-ci, ce taux de taxe n'a pas été rétabli à son niveau antérieur.

Le Livre blanc sur la transition écologique (CGDD-DGT, 2013) reconnaissait sans fard l'insuffisance manifeste de ces taux. Même en admettant la difficulté d'évaluer précisément les coûts externes associés à la pollution de l'air ou à l'usage des infrastructures routières, la présomption est ici également forte que les taux de taxe pratiqués soient très inférieurs aux coûts externes qu'ils sont supposés internaliser.

C'est en vertu d'un diagnostic similaire que, dans son dernier rapport sur l'état environnemental de la France, l'OCDE recommandait déjà d'augmenter les taux des taxes et redevances environnementales (OECD, 2005).

\subsection{De nombreuses niches fiscales nuisibles à l'environnement}

Le système fiscal français comprend de multiples dépenses ou niches fiscales qui sont directement ou indirectement dommageables à l'environnement. Au premier rang de celles-ci figurent les 
nombreuses exonérations accordées par rapport au barème de taxation des énergies fossiles pour des raisons de soutien à certains secteurs (agriculture, BTP, transport routier, taxis, pêche) dont les difficultés ne sont pas forcément liées au prix des carburants, comme le notait Guillaume (2011). Le montant concerné était de près de 6,6 milliards d'euros en 2011 , soit un manque à gagner de $16,5 \%$ des recettes fiscales environnementales de cette année-là... De telles dépenses fiscales atténuent, voire brouillent, le signal-prix envoyé aux consommateurs d'énergies fossiles et contrarient la réorientation souhaitée des comportements. C'est en ce sens qu'elles sont qualifiées de dommageables à l'environnement et comptabilisées comme telles par l'OCDE dans son Inventaire récemment réactualisé (OECD, 2012).

Tableau 2. Principales dépenses fiscales dommageables à l'environnement En M€

\section{Total} 6562

Transports

Exonérations de TIC sur les carburants

- transport aérien commercial (vols internationaux)

- transport aérien commercial (vols domestiques)

Remboursement d'une fraction de la TIC sur le gazole (transport routier de marchandises)

Exonération de TIC sur les produits pétroliers utilisés par les bateaux 278

Exonération de TIC sur les agrocarburants $\left({ }^{*}\right)$

Autres

\begin{tabular}{lc}
\hline Usages sectoriels & 2219 \\
$\begin{array}{l}\text { Taux réduit de TIC sur le gazole non routier (travaux publics, agriculture) } \\
\text { Remboursement d'une fraction de la TIC sur les produits énergétiques } \\
\text { (agriculture) }\end{array}$ & 2080 \\
Autres & 130 \\
\hline $\begin{array}{l}\text { Ménages et Territoires } \\
\begin{array}{l}\text { Exonération de TIC sur le gaz naturel des ménages et des réseaux } \\
\text { de chaleur }\end{array}\end{array}$ & 259 \\
Autres & 253 \\
\hline
\end{tabular}

$\left({ }^{\star}\right)$ Suppression progressive à l'horizon 2016.

Source : CGDD (2013), La fiscalité environnementale en France - États des lieux. 
On peut aussi ranger dans cette catégorie de soutien aux usages de l'énergie, contraire au souhait de promouvoir la sobriété énergétique, les tarifs sociaux de l'électricité et du gaz, dont la création remonte à 2005 pour l'électricité et à 2008 pour le gaz: leur objectif est clairement de lutter contre la précarité énergétique. Leur remplacement par le chèque énergie destiné aux ménages modestes dans la loi «sur la transition énergétique pour une croissance verte » votée en mars 2015, permet de dissocier le montant de l'aide de la consommation effective, ce qui contribue à sortir les consommateurs du rationnement tout en les incitant à la sobriété énergétique. Outre cela, le chèque énergie est versé, sous conditions de ressources, quelle que soit l'énergie utilisée (y compris le bois ou les énergies renouvelables).

La différence de traitement fiscal entre le gazole et l'essence n'est habituellement pas considérée comme une dépense fiscale car elle ne résulte pas d'une exonération ou d'une réduction du taux mais de la fixation séparée de taux de taxe différents sur des produits différents, quoique rendant le même service. Or, le différentiel de taxation en faveur du gazole est en France l'un des plus élevés parmi les pays européens (Andersen et al., 2014). Ainsi, en 2012, le taux implicite de taxe sur l'essence sans plomb, en prenant pour base le contenu énergétique, était de 18,97€/GJ contre $11,90 € / G J$ sur le gazole ${ }^{7}$. Cet avantage fiscal a eu des conséquences importantes sur la composition du parc automobile, donc sur les aspects industriels et compétitifs du secteur, et sur les impacts environnementaux de la circulation automobile.

La part du diesel dans les immatriculations de véhicules particuliers était ainsi encore de $67 \%$ en 2013, en baisse de 6 points depuis 2012, le pic de 77,3 \% datant de 2008 (ADEME, 2014). Au total dans le parc automobile particulier français, la part du diesel est de 75,2\% en 2013. (CGDD, 2014b).

Contrairement à une opinion courante, la différence de taux moyens d'émission de $\mathrm{CO}_{2}$ des véhicules automobiles selon leur motorisation n'est que très légèrement en faveur du diesel, de seulement environ $5 \mathrm{~g} \mathrm{CO}_{2} / \mathrm{km}$ en 2013 (ADEME, 2014), dans les

7. La différence est encore plus forte en ce qui concerne le taux de taxation implicite du carbone puisque la combustion d'un volume de gazole émet $15 \%$ de $\mathrm{CO} 2$ de plus que la combustion du même volume d'essence (CFE, 2013). 
conditions idéales de test des véhicules neufs. En effet, la combustion d'un litre de gazole produit au moins $15 \%$ de $\mathrm{CO}_{2}$ de plus que pour un litre d'essence (CFE, 2013). Mais la consommation de carburant d'un véhicule diesel n'est vraiment plus faible que celle des moteurs à essence que dans les conditions de rendement optimal, atteint seulement après 10 à 15 minutes de circulation en ville. Le diesel est enfin clairement à la source des émissions de polluants atmosphériques puisqu'un véhicule personnel diesel moyen émet à l'échappement de l'ordre de 2 fois plus d'oxydes d'azote et plus de 30 fois plus de particules par kilomètre parcouru qu'un véhicule personnel essence ${ }^{8}$. En 2012, le gazole produisait $89,2 \%$ des $\mathrm{PM}_{10}$, et $91,6 \%$ des $\mathrm{NO}_{\mathrm{x}}$ dus à la circulation routière (CGDD, 2014b).

De façon paradoxale, le dispositif de bonus-malus écologique a un impact écologique mitigé. Certes, il a montré son efficacité pour réduire le taux d'émission moyen de l'ensemble des véhicules en circulation en France puisque le taux d'émission moyen des véhicules neufs est passé de $162 \mathrm{~g} \mathrm{CO}_{2} / \mathrm{km}$ en 2000 à $117 \mathrm{~g}$ en 2012, avec une accélération de la baisse (-32g) à partir de la mise en place du mécanisme de bonus-malus en 2008. La France avait atteint dès 2010 l'objectif du compromis européen visant à réduire le niveau moyen d'émission des véhicules neufs à $130 \mathrm{~g} \mathrm{CO}_{2} / \mathrm{km}$ à l'horizon 2015. Le prochain objectif consiste à ce que $95 \%$ de la flotte automobile émette moins de $95 \mathrm{~g}$ de $\mathrm{CO}_{2} / \mathrm{km}$ en 2021.

Mais sous des dehors d'efficacité environnementale, ce mécanisme est à la source d'un certain nombre d'effets pervers. En effet, la réduction des taux d'émission de $\mathrm{CO}_{2}$ obtenue pour les véhicules neufs mis sur le marché résulte essentiellement de la réduction de leur consommation de carburant. Elle se traduit donc aussi par une baisse du coût d'utilisation au kilomètre parcouru, à prix du carburant donné. D'Haultfœuille et al. (2014) ont montré que sur la période 2008-2009, cette baisse du coût d'utilisation ajoutée à l'avantage-coût procuré par le bonus a provoqué un « effet rebond » sur les émissions totales en raison de l'augmentation des achats de véhicules (les émissions croissant à la fois avec le nombre de kilomètres parcourus mais aussi avec la production de nouveaux

8. http://www.airparif.asso.fr/etat-air/air-et-climat-quelques-chiffres\#sources (consulté le 21/ 12/2014). 
véhicules, elle-même émettrice de $\mathrm{CO}_{2}$ ). Ils suggèrent de réduire le taux d'émission pivot du mécanisme bonus-malus mais aussi de calibrer le mécanisme pour que le « verdissement » de la flotte automobile soit réalisé sans variation de la taille de cette flotte. En 2014, les seuils de déclenchement du bonus et du malus ont été modifiés de façon plus radicale que les années précédentes : le bonus n'étant plus attribué que pour des véhicules émettant moins de $95 \mathrm{gCO}_{2} / \mathrm{km}$ tandis que le malus frappe tous ceux qui émettent plus de $130 \mathrm{gCO}_{2} /$ km (ce qui était le seuil de déclenchement du bonus en 2008). Meurisse et Le Roy (2014) montrent qu'il est possible d'accompagner le mécanisme de bonus-malus d'une taxe sur les carburants pour neutraliser cet effet rebond, sans pour autant annihiler l'effet incitatif à l'achat d'un véhicule moins émetteur.

En outre, le bonus-malus ne prend en compte que le niveau d'émissions de $\mathrm{CO}_{2}$ des véhicules et non les autres pollutions, les émissions de particules par exemple. À taux d'émission de $\mathrm{CO}_{2}$ égal, un véhicule diesel sera ainsi réputé aussi "propre " qu'un véhicule ordinaire. Le caractère incitatif du bonus/malus est donc insuffisant et trop exclusivement orienté vers la lutte contre le changement climatique.

Réviser le principe du bonus/malus afin de le rendre plus incitatif et plus efficace encore supposerait de le différencier selon le type de carburant utilisé et surtout de favoriser bien davantage, en termes relatifs, les véhicules non émetteurs (comme les véhicules électriques ou à hydrogène) ce qui commence à être le cas depuis 2014. Entré en vigueur au le $1^{\mathrm{er}}$ avril 2015, le super-bonus constitué par la prime à la conversion des véhicules diesel de plus de 15 ans pour l'achat d'une voiture électrique (10 000 euros) ou d'une voiture hybride (6 500 euros) va dans ce sens, même s'il ne concerne que la frange la plus polluante du parc automobile.

Enfin, en ce qui concerne la biodiversité, de nombreuses mesures fiscales instituées dans le cadre de certaines politiques publiques entraînent indirectement des externalités négatives (Pelosse et al., 2011), ce qui justifie l'objectif de réduction de ces mesures fixé par la Feuille de route 2012 pour la transition écologique (MEDD, 2014) : c'est le cas des dépenses fiscales contribuant à l'étalement urbain, des mesures prises en faveur de la construction de logements neufs (PTZ neuf, dispositif Scellier), de la construction de hangars et d'entrepôts (abattement sur la taxe 
d'aménagement), de la création de bureaux en île-de-France, ou encore l'absence de paiement du coût des équipements collectifs et des externalités par l'urbanisation périphérique (Sainteny, 2011).

\section{Les difficultés rencontrées aujourd'hui par la fiscalité écologique en France}

Le Livre blanc sur la transition écologique (CGDD-DGT, 2013) plaidait pour une réorientation de la fiscalité écologique, allant même au-delà du rapport intermédiaire du CFE paru mi-2013 et plaçant parmi les priorités de renforcer la fiscalité écologique et rendre plus efficaces les subventions écologiques afin de "mettre en place des signaux de prix écologiques reflétant les enjeux de long terme ». Des tentatives ont été faites, en particulier pour mettre en place une fiscalité carbone, mais elles se soldent soit par des échecs comme en 2009-2010, soit plus récemment par un succès très relatif et encore fragile.

\subsection{Une fiscalité écologique non assumée}

Pour limiter la contribution française aux émissions de gaz à effet de serre, le projet de contribution climat-énergie (CCE), voté en 2009 à une large majorité par le Parlement dans le cadre du PLF 2010, prévoyait une taxation des émissions de dioxyde de carbone, à un taux initial de 17 euros/tonne $\mathrm{CO}_{2}$ en 2010 sur l'ensemble des usages d'énergies fossiles non couverts par le marché européen de permis d'émission négociables. Le rapport Rocard, rendu dans la phase de préparation du projet, préconisait pourtant une montée en charge depuis 32 euros/tonne jusqu'à 100 euros en 2030, en insistant sur l'importance de ne pas décrédibiliser le signal-prix à moyen-terme en adoptant un niveau de départ d'un ordre de grandeur trop faible. Mais l'exemption de la quasi-totalité des entreprises industrielles (93\% des entreprises, car assujetties au SCEQE, ou marché européen de quotas d'émission de GES) a provoqué la censure du Conseil constitutionnel et l'abandon du projet.

La dénomination de contribution climat-énergie mettait l'accent sur le fait que s'acquitter d'une taxe sur le carbone émis revient à verser une contribution au financement du bien public qu'est le climat. En ceci, la CCE affirmait sa nature de taxe d'internalisation d'une externalité par opposition à toute notion d'impôt 
de rendement. Mais, aussitôt rebaptisée «taxe carbone» par l'ensemble des acteurs politiques et économiques, la CCE avait perdu en lisibilité et en acceptabilité pour ne plus être perçue par l'opinion publique que comme un prélèvement supplémentaire.

Quatre ans après cet abandon, et sur proposition du CFE, une nouvelle contribution climat-énergie est entrée en vigueur au $1^{\text {er }}$ avril 2014 au taux de 7 euros/tonne $\mathrm{CO}_{2}$ en 2014 , puis de 14,50 euros en 2015 et qui doit monter à 22 euros en 2016. Sa caractéristique essentielle est sans aucun doute de ne pas être une nouvelle taxe sur une nouvelle assiette mais un nouveau mode de calcul d'une partie d'une taxe existante : c'est une "composante carbone » qui relève les taux de TICPE (taxes intérieures sur la consommation d'énergies fossiles) via une accise carbone. Au contraire de la TVA, qui est une taxe ad valorem, dont le rendement augmente avec le prix hors-taxe des carburants et combustibles, la CCE comme la TICPE dans son ensemble sont insensibles aux variations de prix des produits taxés : à volume de consommation inchangé, leur rendement reste le même et leur poids relatif s'affaiblit en cas de hausse de prix des produits pétroliers. C'est une propriété importante qui permet d'atténuer les effets de la volatilité du prix du baril de brut grâce à l'inertie du prix des carburants à la consommation, déjà forte en raison du poids très fort des taxes (dont la TVA) dans le prix final, qui représentaient, fin 2014, $60 \%$ du prix de l'essence et $52 \%$ du prix du gazole. Par exemple, alors qu'en France les prix du gazole et de l'essence ont retrouvé fin 2014 leur niveau de décembre 2010 en raison de la baisse spectaculaire du prix du pétrole, la baisse est beaucoup plus forte aux États-Unis (en raison d'une hausse du dollar par rapport à l'euro) où les ventes d'automobiles sont immédiatement reparties à la hausse. Ce phénomène fournit une superbe illustration de l'existence de l'élasticité de la demande de carburant au prix : la consommation de carburant est ainsi suffisamment élastique pour que les comportements d'achat soient très différents entre la France et les ÉtatsUnis, étant donné leur très fort différentiel de taxation, mais suffisamment peu pour pouvoir servir de base fiscale à un impôt de rendement...

Comme la TICPE elle-même, sa composante carbone admet encore de nombreuses exonérations et réductions de taux, par exemple pour les départements d'outre-mer (exclusion du champ 
d'application de la TICPE), les agriculteurs (remboursement partiel) ou encore le gaz naturel à l'état gazeux destiné à être utilisé comme carburant (réduction de taxe de $100 \%$ ). Les secteurs des transports aériens et maritimes demeurent "hors-champ » de la TICPE et le secteur du transport routier bénéficie toujours du remboursement de la TICPE, ce qui correspond à une exonération de fait de la composante carbone pour les véhicules de plus de 7,5 tonnes. Enfin, les entreprises électro-intensives soumises à quotas (marché européen EU-ETS) sont exonérées au nom de la préservation de la compétitivité des entreprises (faculté offerte par l'article 17 de la directive 2003/96/CE qui autorise les États membres à appliquer un niveau de taxation allant jusqu'à zéro aux produits énergétiques et à l'électricité lorsqu'ils sont utilisés par des entreprises grandes consommatrices d'énergie). Néanmoins, certaines exonérations antérieures ont été annulées comme celles qui concernaient les consommations des ménages et la disparition à l'horizon 2016 de la réduction de TICPE pour les biocarburants est programmée. El Beze (2014) estime ainsi que "l'assiette de la composante carbone s'avère plus large que celle de la fiscalité carbone de 2009 ».

L'effet de la contribution climat-énergie sur les prix des carburants est très faible. En effet, en 2014, l'introduction de l'assiette carbone a été compensée par une baisse de la composante classique de la TICPE, sauf pour le gaz, très peu taxé jusqu'ici. Puis, à compter de 2015, l'augmentation sera proportionnelle au contenu en $\mathrm{CO}_{2}$ des produits, et sera la plus forte pour le charbon, puis pour le fioul domestique et enfin le gaz. Pour les carburants, l'effet sera très amorti, car les carburants sont soumis à de nombreuses taxes : la TICPE sur le gazole devrait ainsi passer de 42,84 centimes d'euros par litre en 2013 à 46,81 centimes d'euros en 2016 (+9,3\%) et celle sur l'essence de 60,69 centimes d'euros par litre en 2013 à 64,11 centimes d'euros par litre en $2016(+5,7 \%)$.

Elle ne permet pas davantage la correction du différentiel de taxation existant entre diesel et supercarburant, puisqu'à raison d'une réduction de l'écart de 0,55 centime en 3 ans, il faudrait 210 ans pour corriger le différentiel de taxation entre diesel et supercarburant! L'un des premiers avis du CFE, en avril 2013, avait pourtant concerné la dénonciation de cet écart de taxation «à l'inverse de ce que recommanderait la prise en compte des externalités environnementales ». Il rappelait que la défiscalisation du 
diesel est utilisée comme un instrument de soutien sectoriel en dépit de ses effets incitatifs défavorables à l'environnement (CFE, rapport d'étape, 2013).

La CCE version 2014 a derechef été renommée «taxe carbone » mais elle est jusqu'ici passée relativement inaperçue en raison d'une faible médiatisation et surtout de la neutralisation de son impact en 2014, par la baisse d'un montant équivalent de la composante "classique » de la TICPE (non calculée sur le contenu carbone). C'est sans doute son principal défaut que d'avoir été ainsi introduite de façon subreptice, sans véritable pédagogie, et non dans le cadre d'une réforme globale.

\subsection{Une fiscalité contestée}

Force est de constater que la fiscalité écologique n'est pas acceptée en France, ni par l'opinion publique, ni par la classe politique dans sa majorité, ni curieusement par la ministre actuelle de l'environnement. Seule l'absence de médiatisation (aidée par la compensation totale de son effet sur les prix la première année) a en effet permis la mise en place initiale de la CCE. Le ministre de l'époque, Philippe Martin, avait pris soin de préciser que ce dispositif n'est " pas un impôt de plus mais une réforme fiscale » et qu'il n'aura "aucune incidence en termes de pression fiscale sur les Français en $2014 »^{9}$. De même, la porte-parole du gouvernement indiquait «Il ne s'agit pas de créer une nouvelle taxe. Il s'agit simplement de verdir des taxes déjà existantes sur l'énergie » ${ }^{10}$. Le seul point mis en exergue est ainsi l'absence d'effet négatif sur le pouvoir d'achat et non l'utilité de la mesure pour induire des modifications de comportements.

En revanche, le pataquès autour de l'écotaxe poids-lourds, censée faire internaliser par le secteur du transport routier ses externalités, a démontré surtout l'absence de volonté politique face à l'opposition principalement d'un secteur d'activités (le transport routier de marchandises) qui bénéficiait jusqu'ici d'avantages fiscaux (remboursements d'une fraction de la TICPE pour le transport routier). Alors que le principe de l'écotaxe avait été voté par le Parlement avec une quasi-unanimité, les atermoiements qui ont abouti aux reports successifs puis à l'abandon de la mesure,

9. Philippe Martin, Université d'été du PS, La Rochelle, 23 août 2013.

10. Najat Vallaud-Belkacem, Europe 1, 23 août 2013. 
traduisent une faiblesse du gouvernement (et de la classe politique tout entière) vis-à-vis des mouvements sociaux et du lobbying. Or, céder sur les moyens revient, dans ce cas au moins, à céder sur l'objectif.

De façon générale, la fiscalité écologique est critiquée pour deux raisons essentielles : son effet récessif potentiel et son aspect inégalitaire.

\section{Perte de compétitivité et effet récessif}

En augmentant les coûts de production des entreprises, les taxes écologiques provoqueraient des pertes de compétitivité, inciteraient aux délocalisations, et accroîtraient le chômage. C'est la critique principale dans un contexte de croissance faible et de chômage très élevé.

Le risque de pertes de compétitivité doit cependant être relativisé. Certes, les hausses de coût peuvent être importantes pour les entreprises à forte intensité énergétique, mais celles-ci ont souvent des possibilités de substitution qui leur permettent d'atténuer les effets de la hausse de la taxation (Martin et al., 2014). C'est bien le but de ce type de fiscalité. Et pour la compétitivité et les délocalisations, c'est le coût global de production qui importe, dont la fiscalité écologique n'est qu'une faible composante (Bureau et Mougeot, 2004 ; Ederington, 2005). À ceci s'ajoute la crainte de l'inefficacité au niveau planétaire d'une taxe mise en place dans un seul pays, à la fois en raison de la faible contribution de la France aux émissions globales ( $1 \%$ en 2011 mais $11,3 \%$ pour l'UE27) mais aussi des fuites potentielles de carbone. Ces dernières sont avérées : la France ayant réduit ses émissions depuis sa ratification du protocole de Kyoto, l'empreinte carbone de sa demande intérieure est inchangée à 11,6 teq $\mathrm{CO}_{2} /$ hab tandis que ses émissions territoriales ont baissé de 9,5 à 7,7teq $\mathrm{CO}_{2} / \mathrm{hab}$. Cette « délocalisation » des émissions s'explique par la tertiarisation de l'économie et le progrès technique, lequel abaisse les coûts unitaires de production et provoque un effet rebond de la consommation et donc des importations. La crainte de délocalisations d'activités est également forte même si les éléments empiriques ne sont guère probants en ce sens (Grether et al., 2012 ; Marconi, 2012 ; Erdogan, 2013). Ce sont ces craintes qui motivent la réflexion sur des mécanismes d'ajustement fiscal aux frontières (BTA pour Border tax 
adjustment), néanmoins difficiles à mettre en œuvre, qui permettraient de taxer les émissions de $\mathrm{CO}_{2}$ quel que soit leur lieu d'émission (Schubert, 2009).

\section{Perte de pouvoir d'achat, régressivité et aggravation des inégalités}

Comme toute taxe indirecte, une taxe écologique induit une perte de pouvoir d'achat des consommateurs ${ }^{11}$ (effet-revenu), atténuée néanmoins par l'effet-substitution qui les conduit à modifier la composition de leurs achats. Cet effet-substitution est d'autant plus important que l'élasticité de la demande du bien polluant à son prix est élevée. Par conséquent, plus la fiscalité écologique est efficace sur l'environnement, et moins le pouvoir d'achat est affecté ex post.

Au total néanmoins, dans le cas de la mise en place d'une telle taxe sans redistribution de ses recettes fiscales, la baisse de pouvoir d'achat directe concourt à la réduction de l'activité. Une baisse indirecte supplémentaire résulte des pertes d'emploi.

La fiscalité écologique frappe relativement plus les ménages pauvres dont la consommation de produits taxés constitue une part d'autant plus importante de leurs dépenses que leur revenu est faible. En ce sens, la fiscalité écologique est dite régressive (Poterba, 1991 ; Metcalf, 1999 ; Wier et al., 2005 ; Ruiz et Trannoy, 2008). La taxe carbone élève le prix des carburants, donc le coût d'utilisation des automobiles, et celui des combustibles utilisés pour le chauffage des logements et la cuisine. Comme ce sont surtout des dépenses contraintes, et que les ménages les plus pauvres ne disposent souvent pas des marges de manœuvre budgétaires nécessaires pour investir dans un véhicule ou un mode de chauffage moins polluant, leurs possibilités de substitution sont faibles. Ces ménages ont moins de latitude pour échapper à la perte de pouvoir $\mathrm{d}^{\prime} \mathrm{ach}$ at ou pour en réduire la portée ${ }^{12}$. Leur capacité de réduire leurs émissions est donc également limitée. C'est ce que dénonce

11. Ce n'est pas le cas si, au lieu d'élever le niveau des taxes, la taxe écologique passe par un élargissement de la part carbone (comme en 2014, la contribution climat-énergie). Mais alors, sans élévation du prix, il n'y a aucune incitation lisible à réduire la consommation.

12. Des travaux récents relativisent cet aspect régressif de la fiscalité écologique, en tenant compte de la totalité du cycle de vie des agents (Sterner, 2012) ou des effets d'équilibre général (Dissou et Siddiqui, 2014). 
régulièrement Ségolène Royal, ministre de l'écologie depuis avril 2014, comme une manifestation de l'écologie punitive.

\section{Les voies de l'acceptabilité}

Comment faire accepter par les contribuables le principe et la mise en application de la fiscalité écologique ? Au vu de ce qui précède, quelques grands principes se dégagent : redistribuer pour vaincre les craintes de pertes d'emplois et de pouvoir d'achat; le faire habilement pour limiter son impact sur les plus pauvres et démontrer que l'écologie peut tout à fait ne pas être punitive ; expliquer la finalité incitative de la fiscalité écologique par opposition à la logique de rendement ; afficher clairement les objectifs et les moyens mis en œuvre pour surmonter la prévention de l'opinion publique contre l'impôt. Tous ces éléments sont liés.

\subsection{Pédagogie}

Trois points doivent être impérativement mis en exergue pour que les taxes écologiques soient acceptées.

Le premier est que l'objectif doit être compris et partagé : il faut expliquer la nécessité de contribuer à la lutte contre le changement climatique ainsi que la volonté de la France de respecter des engagements pris avec ses partenaires de l'Union européenne devant la communauté internationale, à la mesure de sa responsabilité passée, plus forte que sa contribution actuelle aux émissions mondiales.

Le deuxième est qu'une taxe écologique est définie par son assiette et son taux mais non par l'utilisation de ses recettes : elle taxe des produits polluants à un taux égal (autant que faire se peut, étant donné les incertitudes liées à son estimation) au dommage marginal provoqué par cette pollution afin de réorienter les comportements d'achat vers des produits ou technologies moins polluants. L'efficacité de cet infléchissement des comportements dépend de la sensibilité de la demande au prix et peut donc exiger la mise en place d'un taux élevé. Il faut en outre lutter contre les idées reçues et faire comprendre que les vertus écologiques des taxes en question existent indépendamment de l'utilisation des fonds obtenus, même lorsqu'ils contribuent au financement de dépenses publiques sans rapport avec l'environnement. 
Le troisième point, mais sans doute le plus important, est que la fiscalité écologique n'a pas pour objet premier de procurer un rendement fiscal à l'État. Ce rendement n'est qu'un produit joint de la politique menée. Cela ouvre la porte à toutes les possibilités de redistribution des recettes ainsi obtenues. Les taxes écologiques sont encore trop souvent présentées à l'inverse comme un moyen de financer une dépense. Cela a été le cas pour la CCE présentée fin 2013 comme un moyen de financer en partie, à partir de 2015, le crédit d'impôt compétitivité-emploi. De la même façon, la taxe sur le gazole a été rehaussée de 2 centimes par litre au $1^{\text {er }}$ janvier 2015 pour compenser le manque à gagner de l'écotaxe (PLF 2014) alors même que le gouvernement avait refusé courant 2013 de réaligner progressivement la fiscalité du gazole sur celle de l'essence, comme le proposait le CFE pour des raisons écologiques... Une telle présentation est un moyen sûr de faire naître ou d'entretenir le doute sur l'utilité des taxes écologiques pour l'environnement.

\subsection{Redistribution}

Terkla (1984), Poterba (1993) puis Parry (1995) ont montré que redistribuer les recettes fiscales issues des taxes écologiques permet de réduire leur coût macroéconomique global en réduisant les pertes de pouvoir d'achat des ménages et/ou les hausses de coût unitaire de production des entreprises. Le mode de redistribution dépend de l'objectif principalement visé.

\section{Compensation forfaitaire}

Si l'objectif est de limiter les pertes de pouvoir d'achat des ménages et de contrebalancer l'effet négatif relativement plus fort pour les plus pauvres, le plus simple est de redistribuer aux ménages tout ou partie des recettes fiscales obtenues sous la forme d'une compensation forfaitaire : un " chèque vert » (selon l'exemple de la Suisse). Une telle redistribution est par nature progressive, procurant un supplément de revenu relativement plus important aux revenus les plus faibles. Sa progressivité peut être accentuée si seuls les ménages les plus pauvres reçoivent le chèque vert.

C'était l'un des scénarios discutés par le CFE pour reverser aux ménages une partie des recettes de la composante carbone de la $\mathrm{TICPE}^{13}$. En ne redistribuant cette somme qu'aux ménages situés

13. Soit 700 millions d'euros. La CCE devrait rapporter 4 milliards d'euros à l'État en 2016 dont 2,6 milliards acquittés par les ménages. 
dans les plus bas déciles en termes de revenus, soit 3 à 4 millions de ménages, cela représenterait environ 200 euros par an et par ménage en 2016. Mais les décisions n'ont pas encore été prises sur cette redistribution.

Pour tenir compte de la composition des familles, la compensation forfaitaire peut être versée aux individus plutôt qu'aux ménages : c'est l'esprit de la proposition de taxe carbone pour les États-Unis émanant du Citizens' Climate Lobby qui suggère de verser à chaque adulte un chèque vert du même montant (et la moitié de cette somme à chaque enfant).

\section{Carbon Fee and Dividend ${ }^{14}$ \\ La proposition du Citizens' Climate Lobby}

Le Citizens' Climate Lobby est un groupe de pression écologiste américain dont l'Advisory Board comprend James Hansen, Professeur associé à l'Earth Institute de l'Université Columbia à New-York, bien connu pour ses positions en faveur de la lutte contre le changement climatique et de la taxe carbone.

Il propose un mécanisme reposant sur la combinaison de quatre éléments essentiels : une taxe carbone croissante au cours du temps jusqu'à des niveaux élevés, un ajustement fiscal aux frontières, un mode original de redistribution des recettes fiscales ainsi obtenues et une appellation destinée non seulement à rappeler aux contribuables le sens de l'ensemble du dispositif mais aussi à l'autonomiser par rapport au budget fédéral.

Le prix du carbone initial serait de $10 \$ / \mathrm{tCO}_{2}$ en 2016 , pour augmenter de $10 \$$ chaque année pour atteindre $200 \$ / \mathrm{tCO}_{2}$ afin de permettre en 2050 une réduction des émissions de dioxyde de carbone de $90 \%$ par rapport à 1990 . Ce prix devrait être payé par tous les usages finaux émetteurs de $\mathrm{CO}_{2}$ : ménages, entreprises et services de l'État.

L'ajustement fiscal aux frontières permettrait de taxer les importations provenant de pays n'ayant pas adopté de tarification carbone, tandis que les exportations vers de tels pays seraient détaxées.

La totalité des recettes ainsi dégagées abonderait un fonds, le Carbon Fees Trust fund, qui serait redistribué directement aux ménages, sous la forme d'un chèque mensuel. Chaque adulte recevrait une part et chaque enfant une demi-part. La création du fonds permet de sanctuariser les recettes carbone par rapport au reste du budget et de garantir leur

14. http://citizensclimatelobby.org/carbon-fee-and-dividend/ 
entière redistribution. La totalité du rendement redistribué serait ainsi supérieure à l'impôt acquitté par l'ensemble des ménages : d'où un gain très fort en pouvoir d'achat permettant la relance de l'économie.

La principale originalité de la proposition tient sans doute à sa précision sémantique : le mécanisme fixant un prix au carbone n'est pas une taxe carbone (carbon tax) mais une redevance carbone (carbon fee), la différence étant qu'une taxe abonde le budget national, ce qui suggère que c'est un impôt de rendement, tandis qu'une redevance est une contribution au financement d'un bien ou d'un service dont profite le contribuable. De façon symétrique, le terme de dividende (dividend) versé par un fonds de "placement », (même s'il n'est pas question pour le CCL de placer ces recettes sur les marchés financiers) suggère le retour aujourd'hui d'un investissement sur l'avenir.

Dans le même esprit, un projet de taxation carbone accompagnée d'une redistribution sous forme d'un "dividende carbone » (carbon dividend) a été avancé, pendant l'été 2014, par un représentant démocrate au congrès des États-Unis, Chris Van Hallen, avec le slogan "protéger le revenu des Américains moyens en protégeant la planète pour leurs petits-enfants ».

Pour préserver le caractère incitatif de la taxe, il est essentiel de dissocier la compensation versée aux ménages de leur consommation effective de produits taxés. Il est évidemment possible de redistribuer à l'euro près de façon agrégée, sans que chacun ne reçoive à l'euro près ce qu'il paie !

\section{Verdissement de la fiscalité}

À l'image de la réforme fiscale suédoise du début des années quatre-vingt-dix, il est possible de « verdir la fiscalité » plutôt que de l'alourdir, c'est-à-dire de redistribuer les recettes de la fiscalité écologique en réduisant les taux d'autres impôts ou prélèvements. L'objectif est alors davantage d'augmenter l'efficacité économique en réduisant les distorsions introduites par certains prélèvements obligatoires, selon l'argumentation développée initialement par Baumol et Oates (1988), Pearce (1991) ou Poterba (1993). C'est ainsi Pearce (1991) qui fut le premier à suggérer que la redistribution des recettes de la fiscalité écologique sous la forme de la réduction des taux d'autres impôts distordants pourrait conduire à un double dividende composé d'un premier dividende, environnemental, dû à la réduction des externalités environnementales et d'un second divi- 
dende, d'amélioration du bien-être économique (Goulder, 1995). Ce second dividende peut revêtir les formes complémentaires d'une stimulation de la croissance économique, d'une amélioration du pouvoir d'achat, d'une diminution du chômage, etc. (Ekins, 1997).

Parry (1995) énonce la condition générale garantissant l'existence du dividende économique : il faut que l'effet de recyclage des recettes (revenue-recycling) qui permet de réduire les distorsions fiscales existantes l'emporte sur l'effet d'interaction des taxes (taxinteraction) lequel, par l'érosion mutuelle des bases fiscales, peut au contraire élever le coût brut en bien-être de la taxe environnementale. Mais l'existence même du second dividende réduit en général le premier (l'amélioration de la qualité de l'environnement) car la croissance s'accompagne en particulier de consommation d'énergie. Depuis Bovenberg et De Mooij (1994a) et (1994b), une abondante littérature théorique et empirique s'est attachée à analyser les circonstances et conditions d'obtention d'un double dividende ${ }^{15}$.

Dans la mesure où le double dividende traduit un retour vers l'optimum, plus la situation est sous-optimale, et plus les chances sont grandes qu'une réforme fiscale environnementale neutre budgétairement puisse déboucher sur une situation de double dividende. Celui-ci sera ainsi d'autant plus probable que l'économie est affectée par des imperfections de marché, comme la concurrence imparfaite ou l'existence de chômage (Bovenberg et van der Ploeg, 1996 ; Bovenberg, 1999).

Une condition nécessaire à l'obtention du double dividende est naturellement l'existence d'importantes distorsions fiscales, ce qui est le cas en France et dans les autres pays européens, où les prélèvements sur le travail sont très distordants (EC, 1998 ; ou Fiorito et Padrini, 2001), environ deux fois plus qu'aux États-Unis, au Canada ou au Japon où ce sont les taxes sur le capital qui sont le plus distordantes (Scott, 2007). Chiroleu-Assouline et Fodha (2011) montrent les marges de manœuvre des pays européens en termes de verdissement de leur système fiscal.

Dans le cadre du projet de taxe carbone de 2009, Combet et al. (2010) avaient évalué les possibilités de double dividende permises

15. Chiroleu-Assouline (2001) fournit une revue de cette littérature actualisée depuis celles de Goulder (1995) et Bovenberg (1999). 
par un recyclage des recettes sous la forme de réductions des charges sociales pesant sur les salaires, en se focalisant sur les éventualités de stimulation de la croissance et de l'emploi, comme les études empiriques antérieures (Carraro et al., 1996).

Ainsi, le double dividende n'est-il atteignable que si, simultanément, les marges de manœuvre sont importantes en termes de distorsions fiscales et d'imperfections de marché à corriger et si la fiscalité incitative permet un découplage significatif de la croissance économique et de la pollution (en particulier de la consommation d'énergie fossile). Le double dividende n'est pour autant qu'un bénéfice auxiliaire de la réforme fiscale écologique et ne peut donc que constituer un argument supplémentaire en faveur d'un verdissement de la fiscalité mais certainement pas l'argument essentiel pour mettre en place une taxe écologique. Pour simplifier le résultat à l'extrême, il est toujours moins coûteux, macroéconomiquement, de verdir la fiscalité plutôt que d'ajouter une taxe écologique sans neutraliser ses recettes budgétaires.

L'efficacité économique de la réforme repose sur le remplacement d'une taxe frappant les salariés par une taxe affectant tous les consommateurs, mais sur une base plus étroite que celle de la TVA qui frappe tous les produits de consommation. La correction des distorsions affectant le marché du travail s'opère donc par le biais d'un transfert de charges fiscales vers certaines catégories de ménages: chômeurs, retraités, capitalistes. Le double dividende peut donc tout à fait être obtenu au détriment de celles-ci, l'efficacité économique étant indépendante de l'équité. Par exemple, tous les travaux confirment que l'emploi peut augmenter si la charge fiscale est transférée des travailleurs vers les chômeurs, les résultats dépendant crucialement des caractéristiques du système d'allocations-chômage (Koskela et Schöb, 1999). Aucune réforme ne peut néanmoins être socialement satisfaisante, et politiquement acceptable, si elle accentue les inégalités. Un « chèque vert », même ciblé sur les plus pauvres, ne règle que celle de l'équité et ne procure pas de second dividende. En revanche, ajouter la question de l'équité à celle de l'efficacité économique implique de rechercher des modes de redistribution particuliers.

C'est la motivation principale de Chiroleu-Asssouline et Fodha (2011 et 2014) qui ont démontré la possibilité de compenser la taxe écologique par une baisse progressive des prélèvements sur les 
salaires, c'est-à-dire une baisse uniforme du taux le plus faible du barème progressif (un « chèque vert » étant alors versé aux ménages non imposables) accompagnée d'une hausse de la progressivité. Augmenter la progressivité des prélèvements sur le travail permettrait en effet d'accroître les recettes à redistribuer, ce qui fournit un effet de levier pour renforcer l'acceptabilité de l'introduction de la taxe écologique.

\subsection{Audace et transparence}

Le niveau du taux de taxe joue sans doute un rôle paradoxal dans la compréhension et l'acceptation par les contribuables de la fiscalité écologique. En effet, si la taxe écologique doit internaliser les coûts externes importants (ou au moins aller dans ce sens) provoqués par la pollution et par les émissions de gaz à effet de serre, elle ne peut être efficace que si son taux est d'un ordre de grandeur similaire à celui des dommages provoqués. Des taux élevés permettent à la fois la visibilité du signal-prix et son efficacité sur une base taxable peu élastique. C'est un argument qui devrait être compris de l'opinion publique : en effet, si le problème est important, comment penser le traiter avec des hausses infinitésimales de coûts ? L'introduction de taxes à doses homéopathiques laisse au contraire à penser que la question est moins d'ordre écologique que d'acceptabilité d'un prélèvement supplémentaire. L'argument écologique est alors perçu comme un pur habillage marketing pour une fiscalité de rendement, surtout dans un contexte de finances publiques tendues.

Un taux élevé garantirait également la visibilité de la redistribution des recettes fiscales ainsi obtenues. La compensation significative obtenue par chaque ménage, par rapport à son revenu, ajoutée aux autres aides existantes, pourrait contribuer à l'adoption plus rapide de nouvelles technologies moins polluantes (remplacement de véhicule ou de chaudière).

L'expérience suédoise de l'introduction massive, certes progressive dans le temps, d'une taxe sur le $\mathrm{CO}_{2}$ au début des années quatre-vingt-dix, est un bon exemple à la fois de l'efficacité économique et de l'acceptabilité d'une réforme fiscale d'ensemble ambitieuse. Ce sont en effet $6 \%$ de l'ensemble des prélèvements obligatoires qui ont été remplacés par la taxe $\mathrm{CO}_{2}$ lors d'un verdissement audacieux du système fiscal suédois, avec un taux de taxe 
qui s'élève maintenant à plus de 100 euros par tonne sur les consommations des ménages. Faire preuve d'une audace analogue serait au fond plus convaincant que de continuer à avancer à pas comptés, voire dissimulés. C'est une véritable révolution de notre fiscalité qu'il faut mener.

L'audace indispensable doit être accompagnée de la transparence la plus totale: sur les objectifs, sur la trajectoire de long terme envisagée pour les taux de taxe, et surtout sur l'utilisation des recettes et la logique d'ensemble de la réforme fiscale. En Colombie britannique (Canada) par exemple, les recettes levées grâce à la taxe carbone mise en place en 2008 ne sont pas sanctuarisées comme dans la proposition du CCL évoquée plus haut, mais leur redistribution, votée chaque année comme toute disposition fiscale, est librement consultable sur un site internet ${ }^{16}$ (qui comporte une foire aux questions et permet aux contribuables de s'informer sur la finalité et le fonctionnement de la taxe et surtout sur les modalités adoptées pour en assurer la neutralité budgétaire). Dans une époque de méfiance vis-à-vis du politique, et de croyances conspirationnistes, la transparence est une condition nécessaire de l'acceptabilité d'une réforme de grande ampleur.

\section{Conclusion}

Cet article a démontré que la fiscalité environnementale ne tient pas la place qui devrait être la sienne dans le système fiscal français. La façon dont elle a été progressivement constituée au cours du temps traduit une logique de recherche de rendement fiscal bien plus que d'incitations à modifier les comportements afin de réduire les pollutions ou les émissions de gaz à effet de serre. En cela, elle est fort peu écologique. Différentes tentatives ont été menées dans le passé pour corriger, du moins partiellement, ces défauts, dans le respect du principe pollueur-payeur. Elles ont toutes échoué face à la résistance forte de l'opinion publique et de divers lobbies. La dernière née des taxes écologiques est la CCE entrée en vigueur subrepticement en avril 2014, l'écotaxe poidslourds étant pour sa part, mort-née...

16. http://www.fin.gov.bc.ca/tbs/tp/climate/carbon_tax.htm 
L'analyse des difficultés rencontrées et la comparaison avec des expériences étrangères couronnées de succès offre des perspectives pour réussir enfin en France une réforme fiscale à visée écologique. L'affirmation du principe pollueur-payeur, la fixation de taux de taxe en rapport avec le niveau des dommages environnementaux et la redistribution intégrale des recettes fiscales ainsi obtenues, indépendamment des dépenses en produits taxés, constituent conjointement, mais dans cet ordre, la clé de voûte de la réussite. Le choix des modalités de redistribution dépend des pondérations accordées aux objectifs d'efficacité économique (stimulation de la croissance et de l'emploi) et d'équité (compensation du caractère régressif de la taxation des produits énergétiques). Mais la transparence sur les objectifs, ainsi que des engagements fermes sur une trajectoire de croissance des taux à moyen-long terme, sont tout autant nécessaires.

La mise en place de la contribution climat-énergie en 2014 est un tout premier pas, mais trop timide et mal motivé, dans la direction d'un renforcement du caractère écologique du système fiscal français. Il ne s'agit pas seulement de verdir une fiscalité existante sur l'énergie mais bien plutôt de verdir l'ensemble du système fiscal. La justification de l'instauration de la CCE ne peut pas être le financement du crédit d'impôt compétitivité-emploi mais ses propriétés incitatives à l'adoption de technologies ou de produits moins émetteurs de dioxyde de carbone. Le financement du CICE est seulement l'une des possibilités de recyclage des recettes fiscales ainsi obtenues, qui permettrait éventuellement l'obtention d'un double dividende tandis que les impératifs d'équité sociale conduisent à redistribuer une partie des recettes aux ménages afin de compenser les pertes de pouvoir d'achat des ménages les plus pauvres. Enfin, fixer dès maintenant des taux de taxe plus élevés, pour renforcer la crédibilité écologique de la CCE en profitant de la baisse des cours du pétrole, et jumeler son introduction avec une intensification de la progressivité des prélèvements sur les revenus ferait jouer un effet de levier d'autant plus significatif sur les montants à redistribuer.

Enfin, autant que les actes, les mots ont de l'importance : à l'image de la Colombie britannique où les revenus de la taxe carbone sont redistribués sous la forme de Climate Action Dividends, ou de la proposition du CCL qui insiste sur le caractère contributif 
du prix du carbone, il serait extrêmement utile pédagogiquement d'éviter la dénomination de taxe carbone en France et de s'en tenir au nom de contribution climat-énergie, voire de promouvoir l'intitulé de "dividende carbone » ou de "dividende de la politique climatique » pour les compensations reversées aux ménages.

\section{Références}

ADEME, 2009, Fiscalité comparée de l'énergie et du $\mathrm{CO}_{2}$ en Europe et en France, Stratégies et études.

ADEME, 2014, Évolution du marché, caractéristiques environnementales et techniques - Véhicules particuliers neufs vendus en France, Données et Références.

Andersen M. S., T. Elliott, C. Sherrington, T. Vergunst, S. Ettlinger, L. Elliott et J. Hudson, 2014, Study on Environmental Fiscal Reform Potential in 12 EU Member States, Final Report to DG Environment of the European Commission, Eunomia, Brussels.

Baumol W. J. et W. E. Oates, 1988, The Theory of Environmental Policy, Cambridge University Press, $2^{\text {nd }}$ edition.

Beaumais O. et M. Chiroleu-Assouline, 2002, Économie de l'environnement, Bréal, Paris.

Bovenberg A. L., 1999, « Green Tax Reforms and the Double Dividend: an Updated Reader's Guide », International Tax and Public Finance, $6: 421$ 444.

Bovenberg A. L. et R. A. de Mooij, 1994a, «Environmental Levies and Distortionary Taxation », American Economic Review, 84(4) : 1085-1089.

Bovenberg A. L. et R. A. de Mooij, 1994b, «Environmental Taxes and Labor-Market Distortions », European Journal of Political Economy, 10 : 655-683.

Bovenberg A. L. et F. van der Ploeg, 1996, "Optimal Taxation, Public Goods and Environmental Policy with Involuntary Unemployment », Journal of Public Economics, 62 : 52-83.

Bureau D. et M. Mougeot, 2004, Politiques environnementales et compétitivité, La Documentation française.

Carraro C., M. Galeotti et M. Gallo, 1996, «Environmental Taxation and Unemployment: Some Evidence on the "Double Dividend Hypothesis" in Europe », Journal of Public Economics, 62 : 141-181.

CE Delft/INFRAS, 2008, Handbook on estimation of external costs in the transport sector.

Chiroleu-Assouline M., 2001, «Le double dividende - Les approches théoriques », Revue Française d'Économie, 16 : 119-147. 
Chiroleu-Assouline M., 2011, « La fiscalité environnementale, instrument économique par excellence", Revue Française de Finances Publiques, $114: 17-25$.

Chiroleu-Assouline M. et M. Fodha, 2011, «Verdissement de la fiscalité : à qui profite le double dividende ? », Revue de l'OFCE, 116 : 409-432.

Chiroleu-Assouline M. et M. Fodha, 2014, «From Regressive Pollution Taxes to Progressive Environmental Tax Reforms », European Economic Review, 69 : 126-142, juin, http://dx.doi.org/10.1016/j.euroecorev. 2013.12.006.

Combet E., F. Ghersi, J. C. Hourcade et C. Thubin, 2010, « La fiscalité carbone au risque des enjeux d'équité », Revue française d'économie, 25(2) : 59-91.

Comité pour la Fiscalité Écologique, 2013, Rapport d'étape: Travaux du Comité pour la fiscalité écologique - premier semestre 2013.

Commissariat Général au Développement Durable, 2013, Références - La fiscalité environnementale en France - Un état des lieux.

Commissariat Général au Développement Durable, 2014a, L'économie de l'environnement en 2012, Rapport à la Commission des comptes et de l'économie de l'Environnement.

Commissariat Général au Développement Durable, 2014b, Les comptes des transports en 2013, 51 ${ }^{\mathrm{e}}$ Rapport à la Commission des comptes des transports de la Nation.

Commissariat Général au Développement Durable et Direction Générale du Trésor, 2013, Livre blanc sur le financement de la transition écologique.

Cour des Comptes, 2011, Les prélèvements fiscaux et sociaux en France et en Allemagne, Rapport thématique.

d'Haultfœuille X., P. Givord et X. Boutin, 2014, « The Environmental Effect of Green Taxation: the Case of the French Bonus/Malus », The Economic Journal.

Dissou Y. et M. S. Siddiqui, 2014, "Can Carbon Taxes Be Progressive? », Energy Economics, 42 : 88-100.

Ederington J., A. Levinson et J. Minier, 2005, «Footloose and pollutionfree », Review of Economics and Statistics, 87(1) : 92-99.

El Beze J., 2014, «La réforme de la fiscalité de l'énergie : une extension de la tarification du carbone en France ", Chaire Économie du climat, Policy Brief.

Erdogan A.M., 2013, «Foreign Direct Investment and Environmental Regulations: a Survey », Journal of Economic Surveys.

European Commission, 1998, Environment and Employment: Building a Sustainable Europe, Brussels: EC, DG XI.

European Environment Agency, 2013, Road user charges for heavy goods vehicles $(H G V)$ - Tables with external costs of air pollution. 
Ekins P., 1997, " On the Dividends from Environmental Taxation », in Ecotaxation, T. O'Riordan, ed., Earthscan Publications.

Fiorito R. et F. Padrini, 2001, «Distortionary taxation and labour market performance », Oxford Bulletin of Economics and Statistics, 63(2) : 173-196.

Goulder L. H., 1995, «Environmental Taxation and the "Double Dividend": A Reader's Guide », International Tax and Public Finance, 2 : 157-183.

Grether J. M., N. A. Mathys et J. De Melo, 2012, « Unravelling the Worldwide Pollution Haven Effect», The Journal of International Trade \& Economic Development, 21(1) : 131-162.

Guillaume H., 2011, Rapport du Comité d'évaluation des dépenses fiscales et des niches sociales.

Koskela E. et R. Schöb., 1999, «Alleviating Unemployment: The Case for Green Tax Reforms », European Economic Review, 43 : 1723-1746.

Marconi D., 2012, «Environmental Regulation and Revealed Comparative Advantages in Europe: is China a Pollution Haven? », Review of International Economics, 20(3) : 616-635.

Martin R., L. B. de Preux et U. J. Wagner, 2014, "The impact of a carbon tax on manufacturing: Evidence from microdata », Journal of Public Economics, 117 : 1-14.

Ministère de l'Écologie, du Développement durable et de l'Énergie, 2014, Suivi de la mise en ouvre de la feuille de route 2012 pour la transition écologique.

Metcalf G. E., 1999, «A Distributional Analysis of Green Tax Reforms », National Tax Journal, 52(4) : 655-682.

Meurisse B. et M. Le Roy, 2014, «Towards a Clean Vehicle Fleet: from Households' Valuation of Fuel Efficiency to Policy Implications », FAERE Working Paper, 2014.03.

OECD, 2005, Environmental Performance Review : France, OECD Publishing.

OECD, 2012, Inventory of Estimated Budgetary Support and Tax Expenditures for Fossil Fuels 2013, OECD Publishing, http://dx.doi.org/10.1787/ 9789264187610-en

OECD, 2013, Taxing Energy Use: A Graphical Analysis, OECD Publishing. http://dx.doi.org/10.1787/9789264183933-en.

Parry I. W. H., 1995, « Pollution Taxes and Revenue Recycling », Journal of Environmental Economics and Management, 29(3) : S64-S77.

Pearce D. W., 1991, «The Role of Carbon Taxes in Adjusting To Global Warming ", The Economic Journal, 101 : 938-948.

Pelosse H., L. Winter et alii., 2011, La fiscalité et la mise en œuvre de la nouvelle stratégie pour la biodiversité, Rapport de l'Inspection générale des Finances et du Conseil général de l'environnement et du développement durable.

Pigou A., 1920, The economics of welfare, McMillan\&Co., London. 
Poterba J. M., 1991, « Is the Gasoline Tax Regressive? », Tax Policy and the Economy, 5 : 145-s264.

Poterba J. M., 1993, «Global Warming Policy: A Public Finance Perspective », Journal of Economic Perspectives, 7(4) : 47-63.

Ramsey F. P., 1927, "A Contribution to the Theory of Taxation », The Economic Journal, 47-61.

RICARDO-AEA, 2014, Update of the Handbook on External Costs of Transport - Report for the European Commission, DG MOVE.

Ruiz N. et A. Trannoy, 2008, « Le caractère régressif des taxes indirectes : les enseignements d'un modèle de microsimulation », Économie et Statistique, $413: 21-46$.

Sainteny G., 2011, Les aides publiques dommageables à la biodiversité, Rapport du Centre d'Analyse Stratégique.

Schubert K., 2009, Pour une taxe carbone !, Ed. ENS.

Scott A., 2007, "Optimal Taxation and OECD Labor Taxes », Journal of Monetary Economics, 54(3) : 925-944.

Sterner T., 2012, « Distributional Effects of Taxing Transport Fuel », Energy Policy, $41: 75-83$.

Terkla D., 1984, « The Efficiency Value of Effluent Tax Revenues », Journal of Environmental Economics and Management, 11 : 107-123.

Wier M., K. Birr-Pedersen, H. Jacobsen et J. Klok, 2005, «Are $\mathrm{CO}_{2}$ Taxes Regressive? Evidence from the Danish Experience », Ecological Economics, 52(2) : 239-251.

Withana S., P. ten Brink, A. Illes, S. Nanni et E. Watkins, 2014, Environmental tax reform in Europe: Opportunities for the future, A report by the Institute for European Environmental Policy (IEEP) for the Netherlands Ministry of Infrastructure and the Environment, Final Report, Brussels. 